\title{
Heavy elements in the early Galaxy
}

\author{
Terese Hansen* \\ University of Heidelberg, ZAH, LSW \\ E-mail: thansen@lsw.uni-heidelberg.de \\ Johannes Andersen \\ Niels Bohr Institute, University of Copenhagen \\ E-mail: ja@astro.ku.dk
}

\section{Birgitta Nordtrom}

Niels Bohr Institute, University of Copenhagen

E-mail: birgitta@nbi.dk

\begin{abstract}
The oldest stars in the universe retain to a great extent detailed information on the chemical composition of the interstellar medium at the time of their birth. Hence the earliest phases of Galactic chemical evolution and nucleosynthesis in the early universe can be investigated by means of the old metal-poor stars. For the majority of extremely metal-poor (EMP) stars the element ratios follow a normal pattern, but $10-15 \%$ of the stars are enhanced in heavy neutron-capture ( $r$ - or $s$-processes) elements by large factors, and about $20 \%$ are strongly enriched in carbon. The enhancement of some elements could be the result of highly non-spherical supernova and inefficient mixing in the early interstellar medium. Alternative, they could be due to mass transfer from a former AGB or supernova binary companion that has now evolved to a white dwarf or neutron star. If the latter explanation is true we can detect these stars as long-period binaries. Radial velocity monitoring over a period of $\sim 5$ years of a sample of EMP stars with either $r$-process element and/or carbon enhancement is presented. The results indicate that pure $r$-process and carbon enhancements are not results of mass transfer from a binary companion.
\end{abstract}

XII International Symposium on Nuclei in the Cosmos

August 5-12, 2012

Cairns, Australia

\footnotetext{
*Speaker.
} 


\section{Introduction}

Extremely metal-poor stars should represent the composition of matter in early galaxies. Thus, from detailed abundance analysis of such stars, we can gain insight into the nucleosynthesis and element enrichment of the early interstellar medium. For the majority of EMP stars the elements ratios follow a normal pattern, but $10-15 \%$ of the stars are enhanced in heavy neutron-capture ( $r$ - or $s$-process) elements by large factors, and about $20 \%$ are strongly enriched in carbon [1]. The enhancement of some elements could be the result of highly non-spherical supernovae and inefficient mixing in the early interstellar medium. Alternatively, they could be due to mass transfer from a former AGB or supernova companion binary, that has now evolved to a white dwarf or a neutron star. If the latter explanation is true, we can detect these stars as long-period binaries.

\section{Sample and Method}

The sample contains 40 stars, 17 of which are enhanced in $r$-process elements, eight with moderate enhancement $(0.3<[\mathrm{r} / \mathrm{Fe}]<1.0)$ and nine with the large overabundances $([\mathrm{r} / \mathrm{Fe}]>1.0)$. The remaining 23 stars are carbon enhanced stars (CEMP), of these 12 show signatures of $s$-process elements in their spectra (CEMP-s) while 8 have no such signatures (CEMP-no). The last three stars show both $r$ - and $s$-process signatures (CEMP-r/s). The sample includes CS22892-052 which is enhanced in both carbon and $r$-process elements [6].

Medium-resolution low S/N spectra of the stars have been obtained over a period of five years with the FIES spectrograph at the Nordic Optical Telescope at La Palma. Cross-correlation have been performed to monitor the radial velocity variations of the stars over the period with a precision of $100-300 \mathrm{~m} / \mathrm{s}$. 


\section{Results}

\section{1 r-process enhanced stars}

Among the $17 r$-process enhanced stars three where found to be binaries [5], the remaining 14 show no significant variation in their radial velocity over the five year period. Figure 1 shows the orbit of one of the observed binaries, HE 1044-2509 with a period of 37 days. In all three binary systems found the stars are normal giant branch binaries and there is no sign that the secondary has passed through the AGB stage of evolution or exploded as a supernova, which could have poluted the surface of the primary [4].

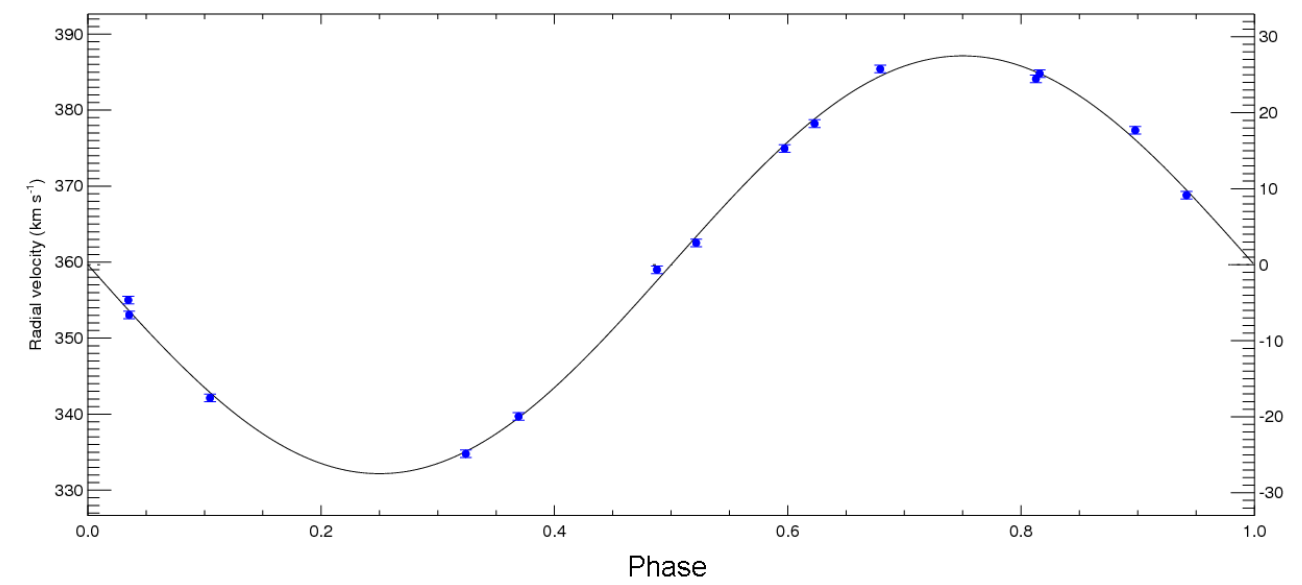

Figure 1: Observed orbit of the $r$-process enhanced star HE 1044-2509, with a period of 37 days.

\subsection{Carbon enhanced stars}

The preliminary results for the carbon enhanced sample show that two out of eight CEMP-no, nine out of twelve CEMP- $s$ and one of the three CEMP- $r / s$ stars are in binary systems. These results are shown in figures 2-4. Figure 2 shows the result of the radial velocity monitoring of a CEMP-no star. The plot clearly shows no significant variation in radial velocities of the star over the five years of measurements. Figure 3 shows the measured radial velocities for a CEMP- $r / s$ star, here there is a clear variation over the five year period, indicating a binary with a very long period. Figure 4 shows an orbit plot of one of the binary CEMP-s star systems. The majority of the CEMP- $s$ stars are found in binary systems, but not all of these are long period binaries. Hence in some orbits there is no room for the secondary to have passed through the AGB phase, where the mass transfer would take place. 


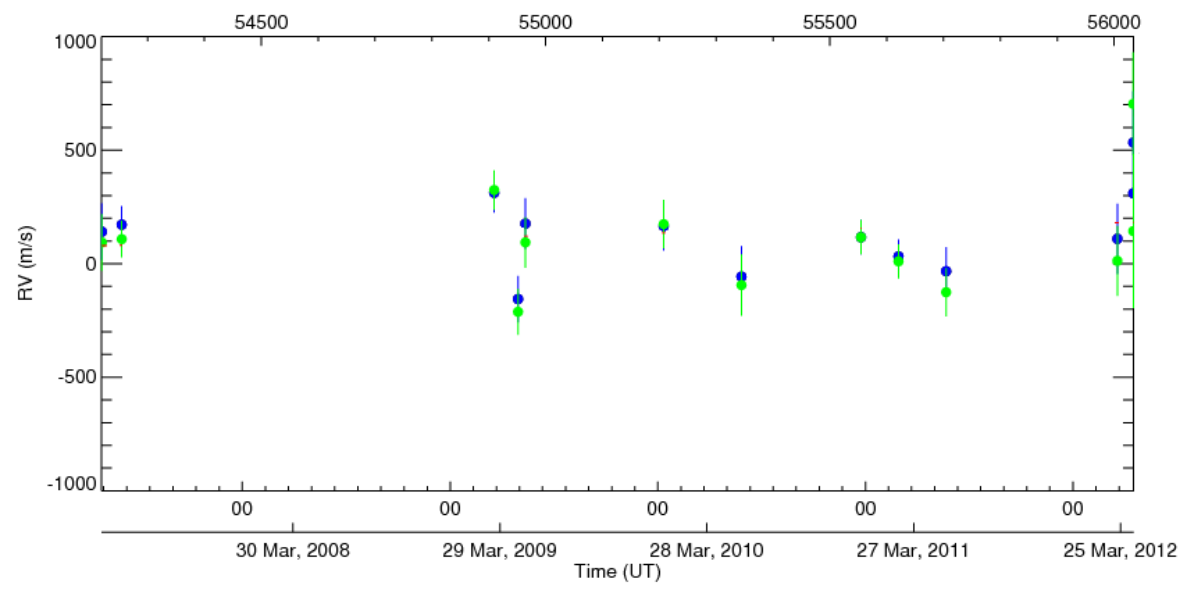

Figure 2: Radial velocity measurements of the CEMP-no star CS 22877-001 over a period of 5 years, $\mathrm{RMS}=179.25 \mathrm{~m} / \mathrm{s}$

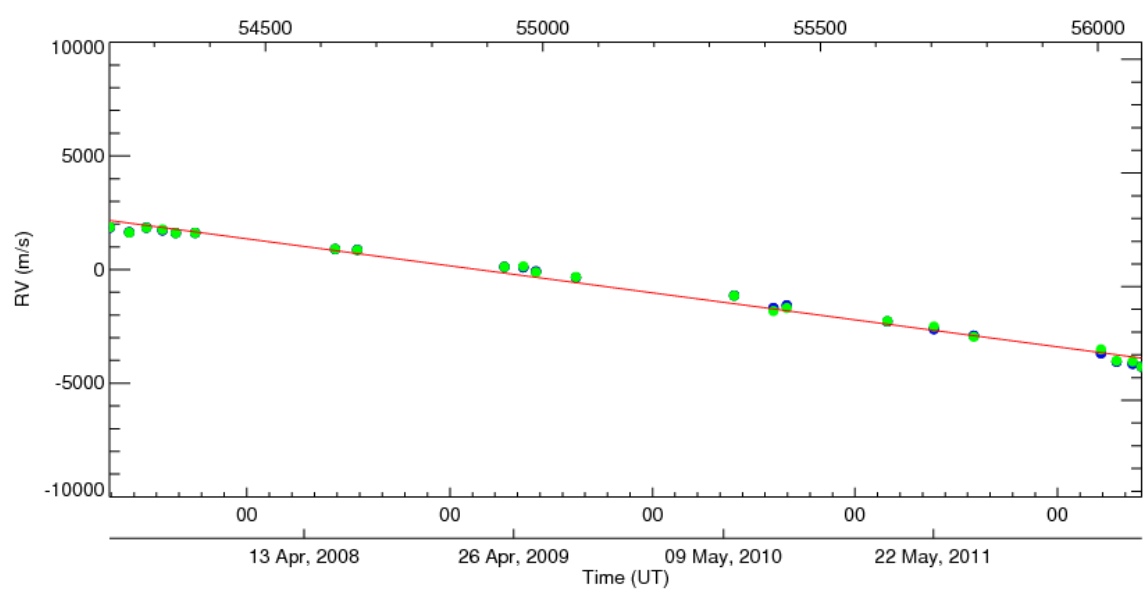

Figure 3: Radial velocity measurements of the CEMP-r/s star LP 625-44 over a period of 5 years. $\mathrm{RMS}=2177.00 \mathrm{~m} / \mathrm{s}$ 


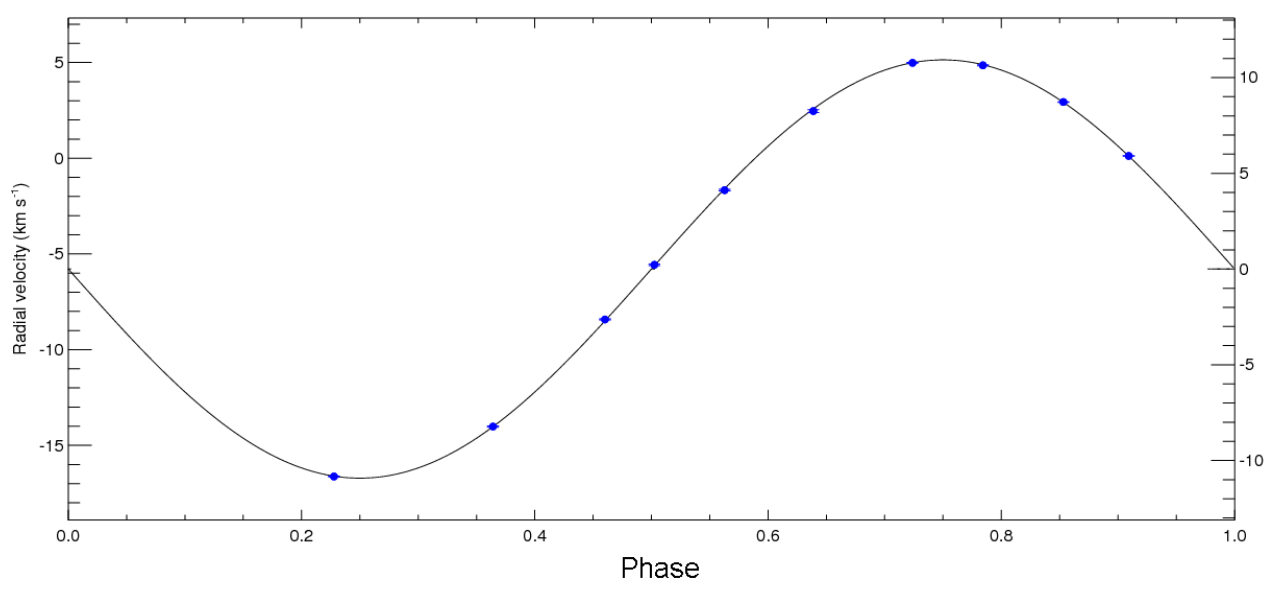

Figure 4: Orbit of CEMP-s star HE 0507-1430 with a period of 446 days.

\section{Conclusions}

The CEMP-no and $r$-process enhanced subsamples are found to have a normal frequency of binary stars, this strongly suggests that the enhancement in carbon and $r$-process elements seen in these stars are not connected with the binary status of the stars. The carbon and $r$-process elements are extrinsic to the stars observed today and were likely injected into the interstellar medium in a collimated manner which make these stars chemical indicators of their formation site in the early Galaxy. The majority of the CEMP-s stars are found to be part of a binary system, hence the origin of the carbon and s-process elements in these stars could be due to mass transfer from an AGB companion, but not all of the binary systems found have long periods with room to accommodate an AGB star.

\section{References}

[1] T. C. Beers \& N. Christlieb, The Discovery and Analysis of Very Metal-Poor Stars in the Galaxy, Ann. rev. A\&A 2005 (43) 531-580.

[2] Hill et al. First stars. I. The extreme r-element rich, iron-poor halo giant CS 31082-001. Implications for the r-process site(s) and radioactive cosmochronology, A\&A 2002 (387) 560-579.

[3] François et al. First stars. VIII. Enrichment of the neutron-capture elements in the early Galaxy, A\&A 2007 (476) 935-950.

[4] Mermilliod, J. C., Statistical Properties of Detected Binary Stars, ASP Conf. Ser. 1996 (90) 95.

[5] Hansen et al. The Binary Frequency of $r$-Process-element-enhanced Metal-poor Stars and Its Implications: Chemical Tagging in the Primitive Halo of the Milky Way, ApJL 2011 (743) L1.

[6] Sneden et al. The Extremely Metal-poor, Neutron Capture-rich Star CS 22892-052: A Comprehensive Abundance Analysis, ApJ 2003 (591) 936-953. 